\title{
Climate change, natural calamities and the triple burden of disease
}

To the Editor - In recent months, there has been an amplified interest in human disease and wellbeing, as well as increased awareness of the vast global disparities in both health risk and care. Despite the ongoing impacts of COVID-19, in general, many developed countries have undergone an epidemiological transition, resulting in a shift in the major types of disease ${ }^{1}$ : the disease pattern has changed to a low burden of communicable and nutrition-deficit diseases and a relatively higher burden of non-communicable diseases (NCDs) and injuries. However, low- and middle-income countries (LMICs) are still in a state of transition, and the burden of communicable diseases in these countries remains considerably high. While ongoing economic growth in LMICs has led to some decreases in these communicable diseases, it has simultaneously increased the burden of NCDs and injuries, giving LMICs a 'triple burden of disease'2. Using the country of Nepal as an example (Box 1), we highlight how climate change can further intensify this triple burden of communicable disease, NCDs and injuries, adding to the disproportionate economic costs of climate change for LMICs.

The environmental determinants of health, such as clean air, safe drinking water and sanitation, are affected by climate change, which can increase communicable diseases and infections, NCDs and injuries. In Nepal, while previously prevalent infectious diseases such as helminth infection, cholera, acute gastroenteritis, tetanus, respiratory infections and tuberculosis have decreased with economic development, emerging and re-emerging infectious diseases such as dengue, chikungunya, influenza (H5N1 and H1N1), scrub typhus, leptospirosis, cryptosporidiosis and malaria have increased $^{3}$. Climate change can lead to geographic expansion of infectious diseases ${ }^{4}$, and has already been implicated in the spread of malaria to hilly and mountainous regions of $\mathrm{Nepal}^{3}$. Moreover, communicable diarrheal diseases and vector-borne diseases such as malaria and dengue are predicted to increase under climate change ${ }^{5}$, and it is estimated that between 2030 and 2050 , climate change will cause 250,000 additional deaths globally per year from communicable and nutritional diseases. Most of these

\author{
Box 1 | Climate change has the potential to increase the triple burden of disease \\ inLMICs such as Nepal
}

Nepal - a developing, seismically active, landlocked country in Southeast Asia - is among the most vulnerable countries in the world due to its fragile landscape, climate-sensitive ecosystem and socioeconomic circumstance. It is placed on the high-risk list in both the Climate Risk Index and the INFORM Risk Index (for humanitarian crises and disasters $)^{18}$. The geographical diversity of Nepal makes it representative of the effects of climate change occurring across a wide range of LMICs. The lowland Terai region is more affected by seasonal flooding due to monsoon rains and a complex river system. By comparison, the hilly region is affected by landslides and debris flows, and the Himalayas are troubled by avalanche and glacial lake outburst floods. These floods, along with the droughts the land experiences, lead to loss of crop production and a food deficit, pushing many people into poverty.

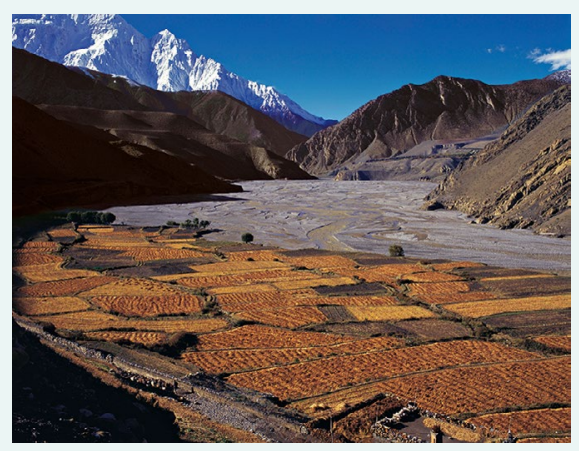

Credit: DGP_travel / Alamy Stock Photo

deaths will occur in developing countries ${ }^{6}$. The situation is worsened by the occurrence of disasters such as floods and landslides, which lead to more clustered disease outbreaks and, under climate change, may occur more frequently and less predictably in space and time ${ }^{7}$. For example, in Nepal, floods and landslides are normally expected in the lowland Terai and hilly regions of Nepal but have also increased in the Himalayan region ${ }^{8}$. Such events are responsible for unexpected direct morbidity and mortality in these ill-equipped regions but can also compound water, sanitation and hygiene (WASH)-related health issues . While economic development has improved facilities for agriculture, tourism and transportation, specific changes in LMICs linked to this development - such as rapid urbanization, agricultural expansion and increased transportation - are also responsible for disease spread across different geographical regions ${ }^{7}$.

In addition to communicable diseases, the interplay of demographic and lifestyle factors in many LMICs has led to an increase in NCDs such as cardiovascular diseases, cancer, chronic respiratory diseases and diabetes $^{1}$. In Nepal, NCDs are responsible for nearly two-thirds of total deaths, and it is projected that by 2040 , about $80 \%$ of total deaths in Nepal will be due to NCDs ${ }^{10}$. Climate change further increases the burden of NCDs through both direct and indirect effects ${ }^{11}$, with changing climatic conditions and increasing temperature linked to acute and chronic respiratory illnesses and cardiovascular diseases ${ }^{12}$, as well as to diseases such as cancer ${ }^{13}$. Climate change significantly affects global food systems and, by decreasing the yield, quality and affordability of food in many LMICs, leads to food insecurity and malnutrition, which is in turn linked to chronic disease ${ }^{14}$. Furthermore, beyond direct impacts, many NCDs may be caused by long-term impacts of infectious diseases, the former of which are expected to increase under climate change.

Climate change may also increase the likelihood of injuries that occur due to hydrometeorological and climatological 
hazards, such as floods, landslides, avalanches, cyclones and wildfires ${ }^{15}$. These types of extreme event produce massive morbidity and mortality, especially in LMICs, as these countries do not have proper preparedness and disaster management plans ${ }^{16}$. In Nepal, between 1971 and 2016, over 26,000 natural disasters were reported, which claimed the lives of over 43,000 Nepalese and left over 83,000 people injured ${ }^{17}$. The trend of disaster occurrence has drastically increased since 2000 , probably as a consequence of increasing hazards due to climate change ${ }^{17}$. Furthermore, economic development with increased transportation in LMICs has led to an increase in road traffic accidents, contributing to injury-related morbidity and mortality ${ }^{10}$.

Many LMICs are based on an agrarian economy, with mild-to-moderate support from industry and tourism, and all of these are vulnerable to the effects of climate change. These effects are thus predicted to cause future instability and massive economic losses in these countries. The additive impact of climate on this state of the triple burden of disease in LMICs is often overlooked, yet it will present a challenge to the development and stability in these countries that must be addressed with concrete, effective, practicable and proactive health and climate policy.

\section{Mitesh Karn (D) $1 \times$ and Muna Sharma ${ }^{2}$ ${ }^{1}$ School of Medicine, Gandaki Medical College Teaching Hospital and Research Center, Pokhara, Nepal. ${ }^{2}$ College of Medicine, Nepalese Army Institute of Health Sciences, Kathmandu, Nepal.

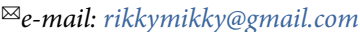

Published online: 30 September 2021 https://doi.org/10.1038/s41558-021-01164-w

\section{References}

1. GBD 2019 Diseases and Injuries Collaborators Lancet 396, 1204-1222 (2020).

2. Marinho, F. M., Soliz, P., Gawryszewski, V. \& Gerger, A. Lancet https://doi.org/10.1016/S0140-6736(13)61343-4 (2013).
3. Rai, S. K. in Infectious Diseases and Nanomedicine III (eds Adhikari, R. \& Thapa, S.) 19-38 (Springer, 2018).

4. Rodó, X. et al. Clim. Change 118, 625-640 (2013).

5. Dhimal, M. et al. Review of Existing Diseases Surveillance System in Nepal from Climate Change Perspective (Nepal Health Research Council, 2019).

6. Climate Change and Health (WHO, 2018); https://go.nature. com/3ClSXIx

7. Wu, X., Tian, H., Zhou, S., Chen, L. \& Xu, B. Sci. China Earth Sci. 57, 189-203 (2014).

8. Eriksson, M. et al. The Changing Himalayas; Impact of Climate Change on Water Resources and Livelihoods in the Greater Himalayas (ICIMOD, 2009); https://go.nature. com/3EBfP95

9. Department for Internal Development Nepal: Climate Change, Health \& WASH (WHO, 2018); https://go.nature.com/3zcY23W 10. Pandey, A. R. et al. PLoS ONE 15, e0243055 (2020).

11. Friel, S. et al. Annu. Rev. Public Health 32, 133-147 (2011). 12. Kinney, P. L. Am. J. Prev. Med. 35, 459-467 (2008).

13. Hiatt, R. A. \& Beyeler, N. Lancet Oncol. 21, E519-E527 (2020).

14. Fischer, G., Shah, M., Tubiello, F. N., \& van Velhuizen, H. Phil. Trans. R. Soc. B 360, 2067-2083 (2005).

15. Sauerborn, R. \& Ebi, K. Glob. Health Action 5, 19295 (2021).

16. Thomas, V. \& López, R. Global Increase in Climate-Related

Disasters (Asian Development Bank, 2015).

17. Shrestha, B. R. Geogr. Base 6, 42-51 (2019).

18. Disaster Risk Reduction in Nepal: Status Report 2019 (UNDRR, 2019).

Competing interests

The authors declare no competing interests. 